Case Report

\title{
Adenomatoid odontogenic tumor - hamartoma or true neoplasm: a case report
}

\author{
Deepti Garg, Sangeeta Palaskar, V. P. Shetty and Anju Bhushan \\ Department of Oral and Maxillofacial Pathology, M. M. College of Dental Sciences and Research, \\ M. M. University, Mullana, India
}

(Received 22 August and accepted 24 December 2008)

\begin{abstract}
Adenomatoid odontogenic tumor (AOT) is an uncommon tumor of odontogenic origin with a relative frequency of $2.2-7.1 \%$. As the histogenesis of AOT is still uncertain, it is sometimes categorized as a hamartomatous lesion rather than a true neoplasm. We report a case of AOT in the right maxillary anterior region in a 20-year-old woman. The tumor showed some unusual and aggressive features that suggested it was a true neoplasm. (J Oral Sci 51, 155-159, 2009)
\end{abstract}

Keywords: adenomatoid odontogenic tumor; hamartoma; true neoplasm.

\section{Introduction}

Adenomatoid odontogenic tumor (AOT) was first reported by Harbitz in 1915 as cystic adamantoma (1). Philipsen and Birn proposed the widely accepted and currently used name adenomatoid odontogenic tumor, a term that was adopted by the first edition of the World Health Organization classification of odontogenic tumors in 1971 (2). However, in 2005 the histologic typing of the WHO defined adenomatoid odontogenic tumor as a tumor composed of odontogenic epithelium presenting a variety of histoarchitectural patterns, embedded in a mature connective tissue stroma, and characterized by slow but progressive growth (3).

Adenomatoid odontogenic tumor has been described as

Correspondence to Dr. Deepti Garg, Department of Oral and Maxillofacial Pathology, M. M. College of Dental Sciences and Research, M. M. University, Mullana-133203, Ambala, Haryana, India

Tel: +91-987-2605676

Fax: +91-172-2584960

E-mail: drdeeptigarg08@gmail.com an uncommon benign odontogenic tumor with a relative frequency of $2.2-7.1 \%$ (4). The tumor appears as an intraoral-extraoral swelling in the maxilla, and is sometimes referred to as "Two-thirds tumor" because it occurs in the maxilla in about $2 / 3$ cases, about $2 / 3$ cases arise in young females, $2 / 3$ cases are associated with an unerupted tooth, and $2 / 3$ affected teeth are canines (5).

As the histogenesis of AOT is still uncertain, there has long been a debate as to whether it represents anomalous hamartomatous growth, or a is true benign neoplasm. This is due, in part, to difficulties with the precise definitions of what seem to be, at least superficially, simple terms and concepts (8). Currently, it is generally accepted to be a true neoplasm (9). Here we describe a case of AOT in a 20year-old woman, with details of some unusual and aggressive clinical, radiographic and histologic features suggesting that it was a true neoplasm.

\section{Case Report}

A 20-year-old woman presented with the chief complaint of swelling on the right side of the face and difficulty in breathing. The patient had first noticed the swelling 1 month prior to presentation, and it had gradually worsened. There was no pain or tenderness associated with the swelling.

\section{Clinical features}

Extraorally, the swelling was located in the maxillary region on the right side lateral to the nose just below the infraorbital margin (Fig. 1), resulting in facial asymmetry. The swelling was irregular in shape and measured approximately $2 \times 3 \mathrm{~cm}$, extending superiorly up to the infraorbital margin, laterally to the zygomatic bone, and inferiorly to the nasolabial fold.

On palpation, the swelling was bony hard in consistency, 
non-tender, and immobile. The surface of the swelling was smooth and the edges were well-defined. Intraorally, the swelling was present in the buccal vestibule on the right side, extending from the distal surface of the lateral incisor to the distal surface of the maxillary 1 st premolar, obliterating the buccal vestibule. The swelling was oval in shape measuring $2 \times 1.5 \mathrm{~cm}$, the overlying mucosa was normal, and the right maxillary canine was missing.

\section{Radiographic features}

Radiographically, OPG showed an ill-defined radiolucent lesion extending anteriorly up to 11 , posteriorly up to 18 , located superiorly near the lower margin of the orbit, associated with an impacted canine extending beyond the cementoenamel junction and inferiorly causing resorption

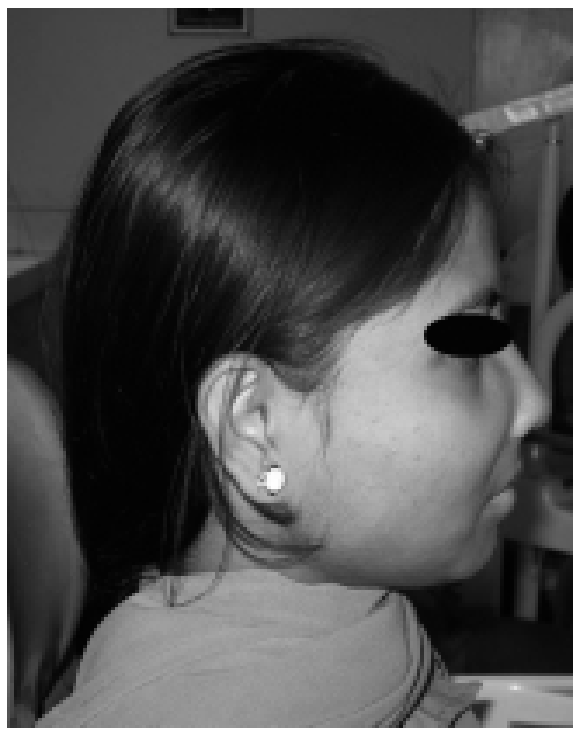

Fig. 1 Swelling in the maxillary region on the right side lateral to the nose just below the infraorbital margin.

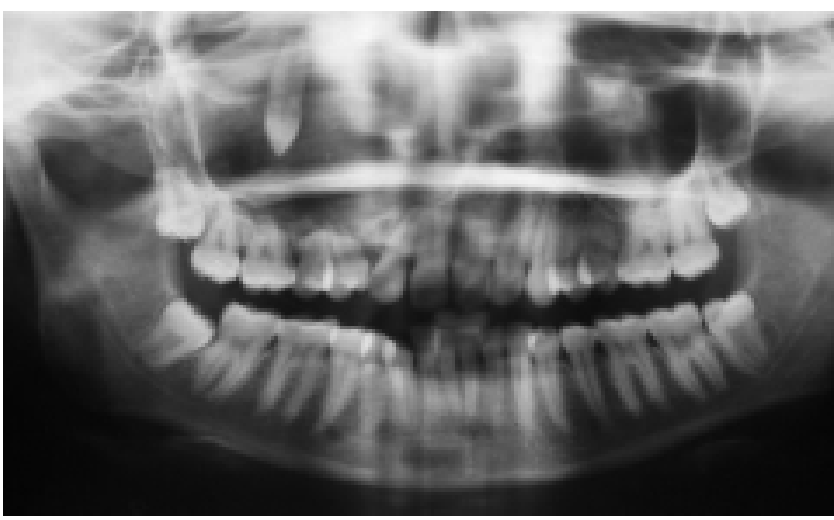

Fig. 2 OPG showing an ill-defined radiolucent lesion causing resorption of the roots of 14,15 and 16 . of the roots of 14, 15 and 16 (Fig. 2). Radioopaque foci were also seen. IOPA showed an ill-defined radiolucent lesion, with foci of radiopacity and resorption of the roots of 14, 15 and 16. Water's view showed an impacted canine near the lower border of the right orbit and haziness in the right maxillary antrum (Fig. 3).

\section{Macroscopic features}

The lesion was enucleated under general anesthesia and sent for histopathological examination. The tumor was brown in color measuring approximately $5 \times 3 \mathrm{~cm}$, and was removed along with the impacted canine. Teeth associated with the lesion and showing resorbed roots were also extracted (Fig. 4).

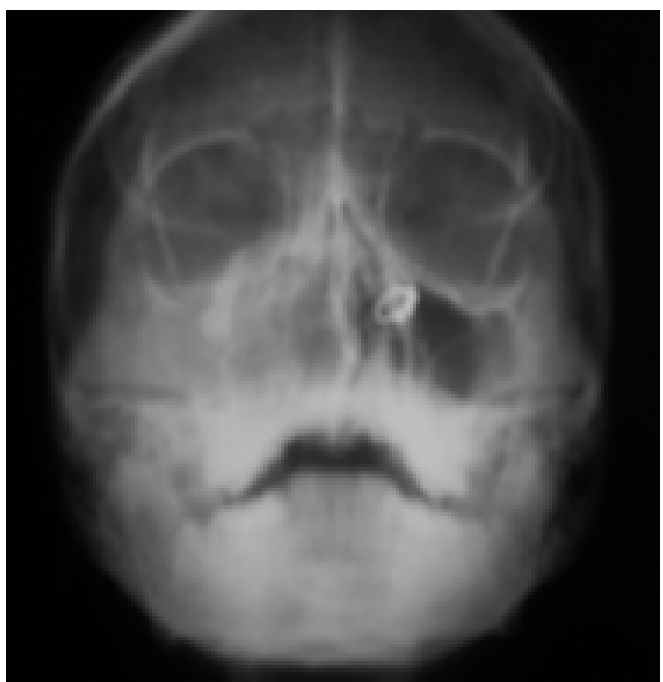

Fig. 3 Water's view showing an impacted canine near the lower border of the right orbit and haziness in the right maxillary antrum.

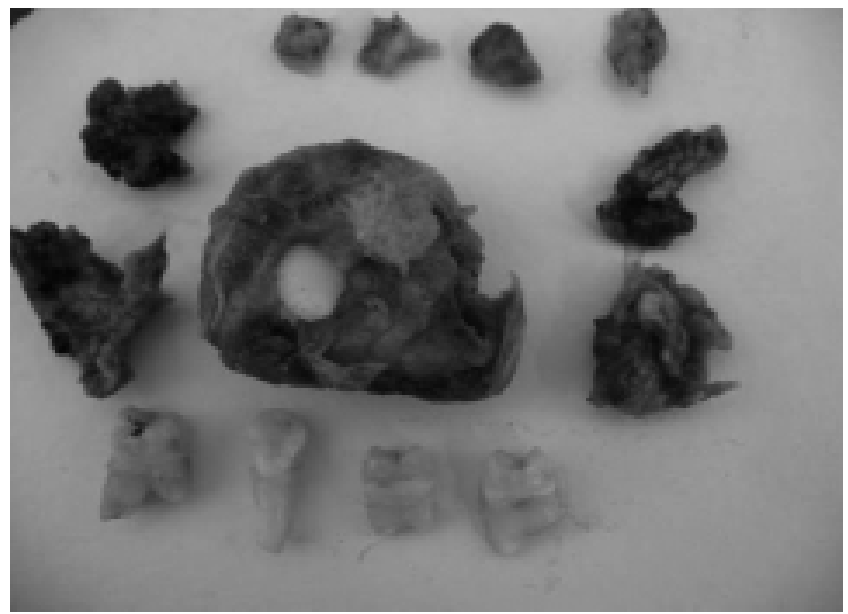

Fig. 4 Macroscopic appearance of the tumor. 


\section{Microscopic features}

Microscopically, the lesion was composed of highly cellular tissue made up of cuboidal and low columnar cells arranged in whorl-like, ductular, ring-like, and ribbonlike patterns (Fig. 5). In some ducts, band of eosinophilic material lined the single layer of cells on the luminal side (Fig. 6). Some areas showed cells arranged in multiple layers. In between each of the layers, a band of eosinophilic material gave a typical rosette pattern to the tumor. Areas of these cells arranged in a solid pattern are also seen. Connective tissue was scanty, with areas showing large blood vessels with extravasated blood. Eosinophilic material was scattered throughout the sections, with small areas of calcification. The overall features confirmed the diagnosis of adenomatoid odontogenic tumor.

\section{Discussion}

AOT is a benign, non-invasive odontogenic lesion showing slow growth. It is generally intraosseous, but can also occur rarely in peripheral locations. AOT is mostly encountered in young patients, especially in the second decade of life, and is uncommon in patients older than 30 years of age. Females are affected by AOT more often than males $(4,6,8)$, with a female: male ratio of $1.9: 1$. This female predilection is even more marked in Asian populations, the highest female incidence being observed in Sri Lanka (3.2:1) and Japan (3:1) (9). The maxilla is the predominant site of occurrence, being almost twice as frequent as that in the mandible, and the anterior part of the jaw is more frequently involved than the posterior

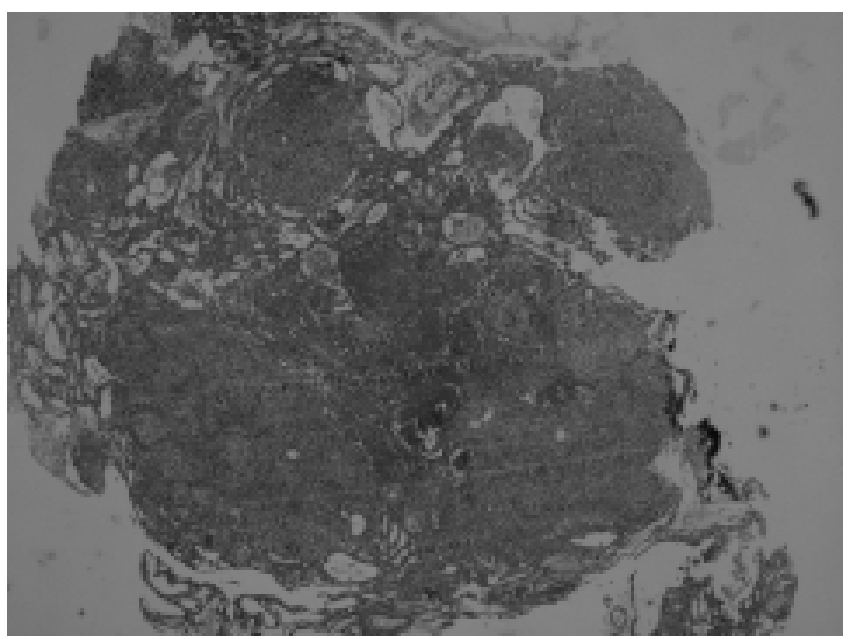

Fig. 5 Microscopically, the tissue appears highly cellular and made up of cuboidal and low columnar cells arranged in whorls, ductular, ring-like, ribbon-like patterns and a rosette pattern $(\times 4)$. part. An unerupted maxillary canine is the tooth most commonly associated with AOT (4). Irregular root resorption is seldom reported (10). Clinically, AOT presents as a slow-growing symptom-free lesion and is frequently discovered during routine radiographic examination (11).

Adenomatoid odontogenic tumor can occur both intraosseously and extraosseously. Intraosseous AOT may be radiographically divided into two types: follicular (or pericoronal) and extrafollicular (or extracoronal). The former is characterized as a well defined unilocular radiolucent lesion surrounding the crown, and is often part of the root of an unerupted tooth. The latter is likewise a well-defined radiolucent lesion, but located between, above, or superimposed upon the root of an unerupted tooth. Minute, variable-shaped radiopacities are frequently found within the lesion. The extraosseous, peripheral, or gingival types of AOT are rarely detected radiographically, but there may be slight erosion of the underlying alveolar bone cortex $(4,6)$.

AOT is usually surrounded by a well developed connective tissue capsule. It may present as a solid mass, a single large cystic space, or as numerous small cystic spaces. The tumor is composed of spindle-shaped or polygonal cells forming sheets and whorled masses in a scant connective tissue stroma. Between the epithelial cells, as well as in the center of the rosette-like structures, are amorphous eosinophilic materials. The characteristic duct-like structures are lined by a single row of columnar epithelial cells, the nuclei of which are polarized away from the central lumen. The lumen may be empty or contain

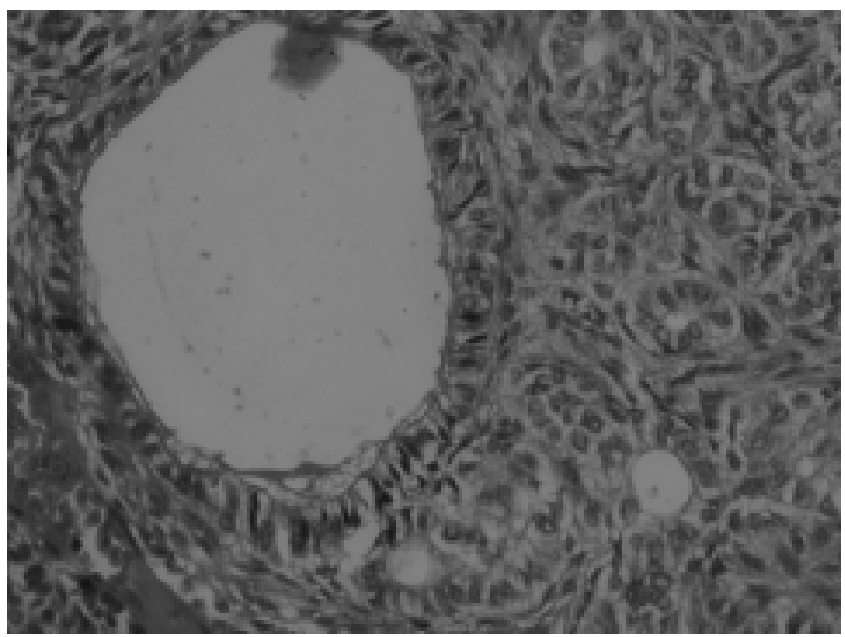

Fig. 6 Tumor cells are arranged in characteristic duct-like structures lined by a single row of columnar epithelial cells, the nuclei of which are polarized away from the central lumen and with a band of amorphous eosinophilic material $(\times 40)$. 
amorphous eosinophilic material. Dystrophic calcification in varying amounts and in different forms is usually encountered in most AOTs within the lumina of the ductlike structures, scattered among epithelial masses or in the stroma $(4,6)$.

The origin of AOT is controversial. However, most authors accept its odontogenic source: it occurs within the tooth-bearing areas of the jaws and is often found in close association with embedded teeth, having cytological features similar to those of the various components of the enamel organ, dental lamina, reduced enamel epithelium and/or their remnants. Some support the idea that the lesion is a developmental outgrowth or hamartoma while others consider it to be a neoplastic growth of odontogenic epithelium. The 1971 WHO classification stated: "it is generally believed that the lesion is not a neoplasm". However, Glickman et al. concluded that "such a controversy is irresolvable because sound arguments can be advanced in favor of and against both hypotheses. The arguments are based on personal bias rather than on scientific evidence" (12).

Some features of AOT suggesting it to be a hamartoma or a true neoplasm have been reported by various authors: Hamartoma

- In 2004, Rick stated that some investigators who prefer to consider AOT to be a hamartoma point to the limited size of most cases and to the lack of recurrence $(4,5)$. Neoplasm

- Authors who consider AOT to be a benign neoplasm believe that the limited size of most cases stems from the fact that they are detected early and removed before the slow-growing tumor reaches a clinically noticeable size (5).

- They also point to the considerable size of some reported cases that had gone undetected and untreated for many years, resulting in facial asymmetry and distortion $(7,13$ 19).

- Also, histologically, the lesion tissue shows greater departure from the arrangement of the normal odontogenic apparatus than would be expected in a developmental anomaly (6).

Interestingly, our present case had some unusual clinical, radiographic and histologic features that distinguished it from most normal types of AOT, and supporting its neoplastic nature. Firstly, AOTs are slow-growing and relatively small in size. However, some large tumors have been reported (7,13-19), and the present case showed unusually rapid growth to more than $3 \mathrm{~cm}$ within 1 month, as reported by the patient. Secondly, radiographic examination usually reveals a well circumscribed radiolucency associated with an impacted tooth, but in the present case the radiolucency was large, ill-defined and associated with root resorption of $14,15,16$, which is quite uncommon. In fact, to our knowledge, only 4 cases of AOT with root resorption have been reported $(9,10,20,21)$. Thirdly, a fibrous capsule is present around the tumor, but this feature was absent in the present case, suggesting its aggressive nature.

Thus, based on currently available evidence and the findings in the present case, we consider adenomatoid odontogenic tumor to be a true neoplasm rather than a hamartoma.

\section{References}

1. Harbitz F (1915) On cystic tumors of the maxilla, and especially on adamantine cystadenomas (adamantomas). Dental cosmos 57, 1081-1093.

2. Philipsen HP, Brin H (1969) The adenomatoid odontogenic tumor, ameloblastic adenomatoid tumor or adeno-ameloblastoma. Acta Pathol Microbiol Scand 75, 375-398.

3. Philipsen HP, Nikai H (2005) Adenomatoid odontogenic tumor. In: Pathology and genetics of head and neck tumors, Barnes L, Eveson JW, Reichart P, Sidransky D eds, IARC Press, Lyon, 304305.

4. Philipsen HP, Reichert PA (1998) Adenomatoid odontogenic tumor: facts and figures. Oral Oncol $35,125-131$.

5. Marx RE, Stern D (2003) Oral and maxillofacial pathology: a rationale for diagnosis and treatment. Quintessence Publishing, Hanover Park, 609-612.

6. Rick GM (2004) Adenomatoid odontogenic tumor. Oral Maxillofac Surg Clin North Am 16, 333-354.

7. Lee JK, Lee KB, Hwang BN (2000) Adenomatoid odontogenic tumor: a case report. J Oral Maxillofac Surg 58, 1161-1164.

8. Swasdison S, Dhanuthai K, Jainkittivong A, Philipsen HP (2008) Adenomatoid odontogenic tumors: an analysis of 67 cases in a Thai population. Oral Surg Oral Med Oral Pathol Oral Radiol Endod 105, 210-215.

9. Nigam S, Gupta SK, Chaturvedi KU (2005) Adenomatoid odontogenic tumor - a rare cause of jaw swelling. Braz Dent J 16, 251-253.

10. Nomura M, Tanimoto K, Takata T, Shimosato T (1992) Mandibular adenomatoid odontogenic tumor with unusual clinicopathologic features. J Oral Maxillofac Surg 50, 282-285.

11. Neville BW, Damm DD, Allen CM, Bouquot JE (2002) Oral and maxillofacial pathology. 2nd ed, WB Saunders, Philadelphia, 621-623. 
12. Philipsen HP, Reichert PA, Zhang KH, Nikai H, Yu QX (1991) Adenomatoid odontogenic tumor: biologic profile based on 499 cases. J Oral Pathol Med 20, 149-158.

13. Raubenheimer EJ, Seeliger JE, van Heerden WF, Dreyer AF (1991) Adenomatoid odontogenic tumor: a report of two large lesions. Dentomaxillofac Radiol 20, 43-45.

14. Layton SA (1992) Adenomatoid odontogenic tumor. Report of an unusual lesion in the posterior maxilla. Dentomaxillofac Radiol 21, 50-52.

15. Geist SM, Mallon HL (1995) Adenomatoid odontogenic tumor: report of an unusually large lesion in the mandible. J Oral Maxillofac Surg 53, 714-717.

16. Bulut E, Tasar F, Akkocaoglu M, Ruacan S (2001) An adenomatoid odontogenic tumor with unusual clinical features. J Oral Sci 43, 283-286.
17. Takahashi K, Yoshino T, Hashimoto S (2001) Unusually large cystic adenomatoid odontogenic tumor of the maxilla: case report. Int J Oral Maxillofac Surg 30, 173-175.

18. Olgaç V, Kögseoğlu BG, Kasapoğlu C (2003) Adenomatoid odontogenic tumor: a report of an unusual maxillary lesion. Quintessence Int 34, 686688.

19. Shetty K, Vastardis S, Giannini P (2005) Management of an unusually large adenomatoid odontogenic tumor. Oral Oncology Extra 41, 316318.

20. Dayi E, Gürbüz G, Bilge OM, Ciftcioğlu MA (1997) Adenomatoid odontogenic tumor: case report and review of the literature. Aust Dent J 42, 315-318.

21. Chuan-Xiang Z, Yan G (2007) Adenomatoid odontogenic tumor: a report of a rare case with recurrence. J Oral Pathol Med 36, 440-443. 